\title{
Fluorescence Imaging in Human Identity Testing
}

\author{
J. Worley, S. Lee, M.S. Ma ${ }^{1}$, A. Eisenberg ${ }^{2}$, H.-Y. Chen ${ }^{2}$ and E. Mansfield ${ }^{2}$ \\ Molecular Dynamics, Sunnyvale, ${ }^{1}$ California Department of Justice DNA Lab, Berkeley, \\ CA, ${ }^{2}$ University of North Texas Health Sciences Center, Fort Worth, TX, USA
}

BioTechniques 23:148-153 (July 1997)

\begin{abstract}
We have investigated the use of fluorescence detection and the FluorImager ${ }^{\circledR}$ SI System (Molecular Dynamics) for analyzing a comprehensive set of human DNA typing tests. We used an alkaline phosphatase-conjugated $\mathrm{YNH}_{24}$ oligonucleotide probe to the repeat-containing D2S44 locus to detect both alleles in $50 \mathrm{ng}$ of human genomic DNA (0.025 amol) by Southern hybridization using a chemifluorescent substrate. We used a similar approach to quantify human DNA using an enzyme-conjugated oligonucleotide probe to the D17Z1 locus. Both fluorescent nucleic acid gel staining and direct fluorescent labeling methods were tested to detect PCR-based D1S80 and short tandem repeat (STR) multiplex allele profiles. The fluorescent staining method sensitively detected these allelic profiles in both denaturing and non-denaturing acrylamide gels using a simple, 10-min procedure. Fluorescent primers eliminate the doublet band patterns often seen with staining methods, which label both strands of the amplified products. This complicates interpretation of STR typing tests. Only one primer for each locus is labeled, so only one strand of the DNA product is detected. Fluorescein end-labeled primers were used in multiplex PCR to amplify, detect and type STRs.
\end{abstract}

\section{INTRODUCTION}

A wide variety of genetic typing tests are currently used in human identification applications for forensic analysis, paternity testing, missing persons identification and immigration documentation. Current casework and research applications include analysis of blots for DNA quantitation and restriction fragment length polymorphisms (RFLP) and gels for polymerase chain reaction (PCR)-based tests such as D1S80 (7) or short tandem repeat (STR) locus analysis (4). Nonradioactive detection methods such as chemiluminescence and DNA silver staining have reduced the need for radioactivity $(1,3,12)$. Fluorescence detection, however, offers excellent sensitivity and a greater dynamic range than these other detection methods $(10,11)$. We have therefore investigated the use of fluorescence detection and analysis using the FluorImager ${ }^{\circledR}$ SI (Molecular Dynamics, Sunnyvale, CA, USA) in human DNA typing applications.

DNA isolated from forensic evidentiary material may contain bacterial or fungal DNA. Accurate quantitation of human DNA in the mixture is necessary, since the amount of human DNA in the sample determines in part which genetic tests are run. This type of quantitation requires a species-specific probe, such as the oligonucleotide probe to the highly repetitive $\alpha$-satellite DNA at the D17Z1 locus (16). This probe can be used to accurately estimate the amount of human DNA in dot- or slot-blotted samples without cross-reactivity from other, non-primate species.

RFLP analysis is currently the most widely used genetic typing method in forensic science. Allelic size variation is the foundation of this method of genetic typing. A Southern blot (14) of genomic DNA samples is probed with DNA that is homologous to one of several highly polymorphic loci. These loci contain a variable number of tandemly repeated $30-80$ bp elements, and consequently, the alleles can vary significantly in size (17). 
PCR amplification has emerged as a very powerful technique for genetic typing. Loci with short (2-16 bp) tandem repeats can be amplified from very small amounts of template, and the products are easily separated on polyacrylamide gels. For example, the D1S80 locus contains a 16-base repeat segment with numerous alleles and a high degree of polymorphism in the human population (7). STR loci generally have fewer alleles, but their shorter repeat size (3-7 bp) allows several loci to be amplified and electrophoresed together, providing a wealth of discriminating genetic information from a single PCR $(4,15)$.

In this paper, we describe the use of the FluorImager SI Fluorescent Scanning system for human identity testing. We demonstrate fluorescent detection methods for sensitive analysis of human DNA on blots and gels. Analytical software improves accuracy in analyzing and quantitating the digital images of each genetic test.

\section{MATERIALS AND METHODS}

\section{FluorImager SI Fluorescent Scanning System}

The FluorImager system rapidly scans and analyzes fluorescently labeled samples in agarose or acrylamide gels, blots, microplates or thin-layer chromatography (TLC) plates. Samples are excited with a $4 \mathrm{~mW}, 488-\mathrm{nm}$, argon-ion laser beam. Fluorescent light emitted by the sample is captured by a fiber-optic collector, amplified with a photomultiplier tube (PMT) (8) and coverted to a digital image. The sample may be scanned with 100 - or $200-\mu \mathrm{m}$ resolution, using either normal or high sensitivity scan mode. The high sensitivity scan mode reduces noise in the image, allowing more sensitive detection of signal in low-background applications (i.e., gelbased). Band-pass filters are used to optimize collection of emitted fluorescent light signals.

The images are viewed and analyzed for fragment size and band quantitation using either ImageQuant ${ }^{\mathrm{TM}}$ or DNA Fragment Analysis software (both from Molecular Dynamics).

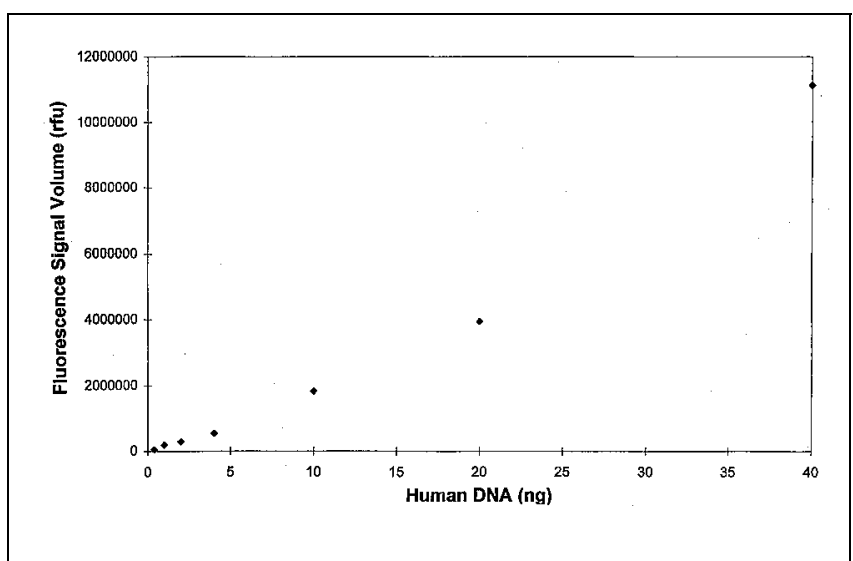

Figure 1. DNA quantitation blot standard curve. A human DNA quantitation slot blot was prepared according to the protocol provided with the ACES Human DNA Quantitation Kit, using $1 \mathrm{mM}$ AttoPhos in place of the chemiluminescent substrate. The blot included a dilution series of human DNA standards ranging from $40-0.2 \mathrm{ng}$ and 22 paternity DNA samples. The signal from each standard was quantitated using ImageQuant analysis software. The signal is linear from $0.4 \mathrm{ng}$ to $20 \mathrm{ng}$ of DNA. All of the unknown samples fell in this range.
These programs enhance viewing of especially weak or intense samples with a gray-scale adjustment feature, and bands are easily quantitated and sized. The results are transferred to Microsoft ${ }^{\circledR}$ Excel $^{\circledR}$ (Microsoft, Redmond, WA, USA) for graphical or statistical interpretation. The digital images are printed on a Model HP-4M Laserprinter (Hewlett-Packard, Palo Alto, CA, USA). Unless otherwise noted, normal sensitivity scan-mode and $200-\mu \mathrm{m}$ resolution scan settings were used in these experiments. The band-pass emission filters and PMT voltage settings are noted for each experiment.

\section{DNA Quantitation Blot}

Paternity casework DNA samples were extracted from liquid blood or dried blood stains using a nonorganic isolation procedure (6). A dilution series of human DNA standard (K562 DNA) and $2 \mu \mathrm{L}$ of each sample were blotted onto Biodyne ${ }^{\circledR}$ A Nylon Membrane (Pall Corporation, Glen Cove, NY, USA) and hybridized according to the protocol in the ACESTM $^{\text {TM }}$.0 Human DNA Quantitation Kit (Life Technologies, Gaithersburg, MD, USA). Following stringency washes, the membrane was heat-sealed in a development bag with 25 $\mu \mathrm{L} / \mathrm{cm}^{2}$ of $1 \mathrm{mM}$ AttoPhos ${ }^{\mathrm{TM}}$ Solution (JBL Scientific, San Luis Obispo, CA, USA) and incubated overnight at room

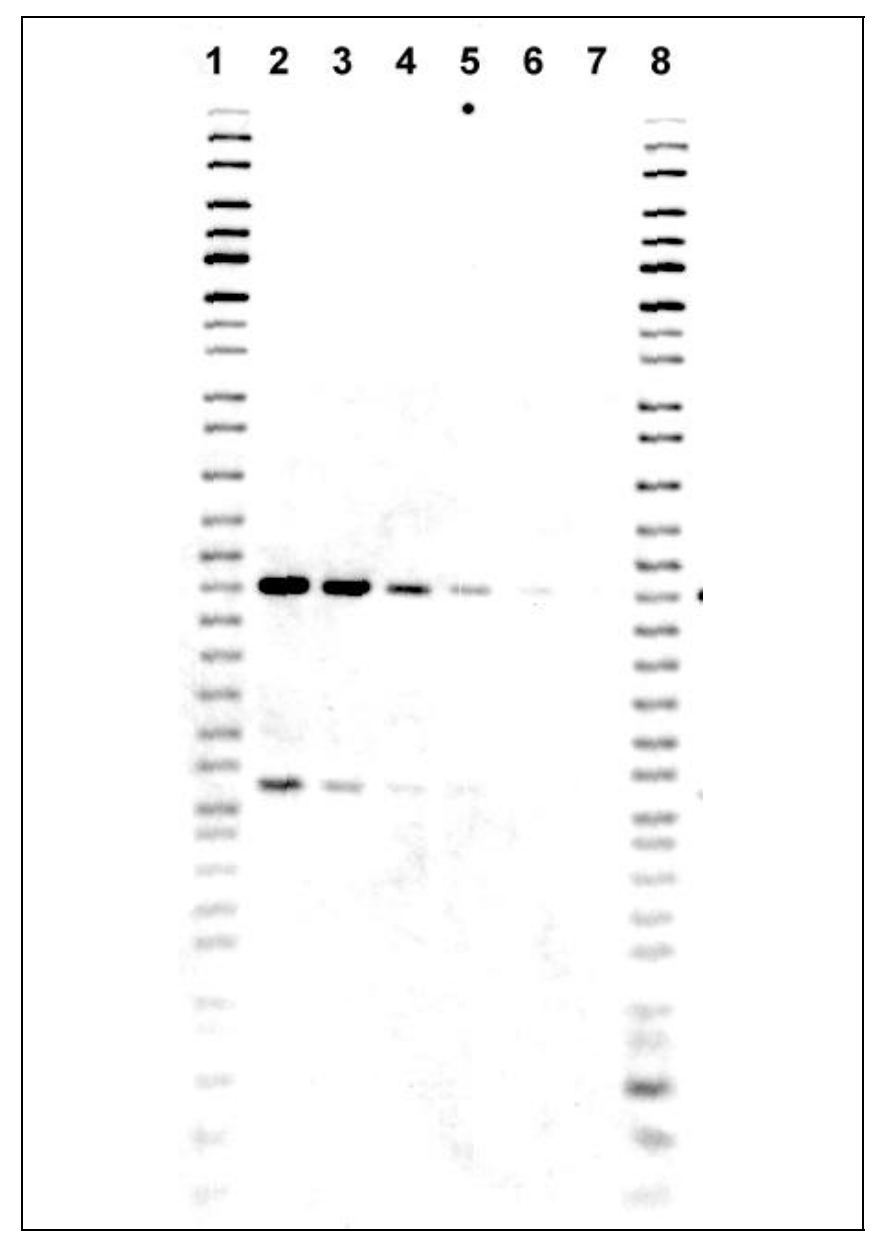

Figure 2. Fluorescent RFLP detection using AttoPhos development. This blot was probed with two alkaline phosphatase conjugated oligonucleotides, one specific for the molecular weight ladder and the second specific for the YNH24 (D2S44) locus. The hybridized blot was incubated overnight with chemifluorescent substrate. Lanes 1 and 8 contain the molecular weight ladder. Lanes 2-7 contain 500, 250, 100, 50, 25 and $10 \mathrm{ng}$ of HaeIII-digested K562 DNA, respectively. 
temperature. The developed membrane was scanned using a PMT voltage setting of $575 \mathrm{~V}$ and a 570DF30 band-pass emission filter (Molecular Dynamics).

\section{Fluorescent RFLP Detection}

A Southern blot of HaeIII-digested human DNA and DNA molecular weight ladder (ACES DNA Analysis Marker System; Life Technologies) was prepared by an alkaline transfer method (5) onto Biodyne A membrane. The baked and UV cross-linked blot was washed for $1 \mathrm{~h}$ in $250 \mathrm{~mL}$ of $2 \times$ standard saline citrate (SSC), $0.1 \%$ sodium dodecyl sulfate (SDS) at $55^{\circ} \mathrm{C}$ to reduce ambient alkaline phosphatase contamination. The washed membranes were then prehybridized $20 \mathrm{~min}$ in $80 \mu \mathrm{L} / \mathrm{cm}^{2}$ of ACES 2.0 Hybridization Solution at $55^{\circ} \mathrm{C}$. This solution was replaced with $50 \mu \mathrm{L} / \mathrm{cm}^{2}$ of ACES $2.0 \mathrm{Hy}-$ bridization Solution containing $0.5-1.0 \mu \mathrm{L} / \mathrm{cm}^{2}$ each of two alkaline phosphatase-conjugated oligonucleotide probes [ACES DNA Analysis Marker System probe and the YNH24 (D2S44) probe (Promega, Madison, WI, USA)] and incubated at $55^{\circ} \mathrm{C}$ for $20 \mathrm{~min}$. The blot was then washed twice for 15 min each in $1.0 \mu \mathrm{L} / \mathrm{cm}^{2}$ of ACES 2.0 Wash Buffer 1 at $55^{\circ} \mathrm{C}$. Two final 15-min washes were conducted at room temperature using $1.0 \mathrm{~mL} / \mathrm{cm}^{2}$ of ACES 2.0 Final Wash Buffer. The membrane was incubated for $5 \mathrm{~min}$ in $16 \mu \mathrm{L} / \mathrm{cm}^{2}$ of $1 \mathrm{mM}$ AttoPhos ${ }^{\mathrm{TM}}$ Solution and heat-sealed in a development bag. The membrane was allowed to develop at $37^{\circ} \mathrm{C}$ for $4-24 \mathrm{~h}$ and scanned using a PMT voltage setting of $575 \mathrm{~V}$ and a 570DF30 band-pass emission filter.

After scanning, blots were stripped by washing for $1 \mathrm{~h}$ in $250 \mathrm{~mL}$ of $55 \%$ formamide, $2 \times$ sodium chloride sodium phosphate EDTA (SSPE), $0.5 \%$ SDS at $65^{\circ} \mathrm{C}$. The stripped blots were then washed for $15 \mathrm{~min}$ in $2 \times \mathrm{SSPE}, 0.5 \%$ SDS at $65^{\circ} \mathrm{C}$. The blot was re-scanned using the same parameters to confirm stripping and allowed to air-dry for storage and reprobing.

\section{SYBR ${ }^{\circledR}$ Green I Staining in D1S80 Typing}

DNA samples were PCR-amplified at the D1S80 locus according to the manufacturer's recommended protocol (PerkinElmer, Norwalk, CT, USA). The 31.8- $\times 19.5-\mathrm{cm} 8 \%$ acrylamide gel (29:1 acrylamide:bis) was prepared with 7\% glycerol in a Tris-sulfate buffer (100 mM Tris-sulfate, $\mathrm{pH} 4.5$, $1 \mathrm{mM}$ EDTA). Before casting the gel, the shorter electrophoresis plate was treated with $3 \mathrm{~mL}$ Gel Slick ${ }^{\mathrm{TM}}$ (AT Biochem, Malvern, PA, USA). The gel was not pre-electrophoresed. After loading, the gel was electrophoresed in $1 \times$ TBE running buffer (100 mM Tris, $90 \mathrm{mM}$ boric acid, $1 \mathrm{mM}$ EDTA, pH 8.3) for $5 \mathrm{~h}$ at $500 \mathrm{~V}$. The gel plates were separated, and the gel was stained with a $15 \mathrm{~mL}$ overlay of a 1:10000 dilution of SYBR ${ }^{\circledR}$ Green I (Molecular Probes, Eugene, OR, USA) for $10 \mathrm{~min}$. Excess stain was drained off the gel, and the wet gel was scanned using a PMT voltage setting of 600 $\mathrm{V}$ and a 530DF30 band-pass emission filter (Molecular Dynamics).

\section{Fluorescent Multiplex STR Typing Using End-Labeled Primer Sets}

Four STR loci (CSF1PO, TPOX, TH01 and vWA) were amplified in a multiplex PCR using a Model 480 Thermal Cycler (Perkin-Elmer) with the GenePrint ${ }^{\mathrm{TM}}$ Fluorescent STR Multiplex System (Promega) according to the protocols of the manufacturer. Samples were amplified from 5-10 ng of extracted DNA. Bovine serum albumin (BSA; $1.5 \mu \mathrm{g}$ per reaction) was added to some reactions to improve yield. Amplified products or allelic ladder standards $(2.5 \mu \mathrm{L})$ were combined with loading buffer and heat-denatured. A 5- $\mu \mathrm{L}$ aliquot of each sample was loaded and separated on a 31.8- $\times$ $19.5-\mathrm{cm} 6 \%$ denaturing polyacrylamide gel (37.5:1 acrylamide:bis, $7 \mathrm{M}$ urea, $0.5 \times \mathrm{TBE}$ ). The gel was electrophoresed in a $0.5 \times$ TBE buffer system for $2 \mathrm{~h}$ at $40 \mathrm{~W}$ constant power. Gel plates were cleaned with water and scanned in high sensitivity scan mode using a PMT voltage setting of $900 \mathrm{~V}$ and a 530DF30 band-pass emission filter.

\section{RESULTS}

\section{Human DNA Quantitation}

Small aliquots of each blood stain DNA isolate (less than $3 \%$ of the total extract) were analyzed using the ACES Human DNA Quantitation System with a chemifluorescent substrate to determine the quantity of human DNA in each test sample (data not shown). Fluorescence intensity of the human genomic DNA standards (0.2-40 ng K562 DNA) were quantitated using ImageQuant, and a standard curve was generated (Figure 1). The signal intensity is linear from 0.4-20 ng of DNA. This curve was used to calculate the DNA concentration of the test samples. The concentrations of all test samples fell within this linear range.

\section{RFLP Detection}

The blot includes the ACES DNA Analysis Marker System standard and a dilution series of HaeIII-digested genomic DNA isolated from the K562 human cell line. After overnight development with a chemifluorescent substrate, both alleles of D2S44 were detected in as little as $50 \mathrm{ng}$ of total genomic DNA (Figure 2). The direct enzyme-conjugated oligonucleotide probes required only 10-40-min hybridization times

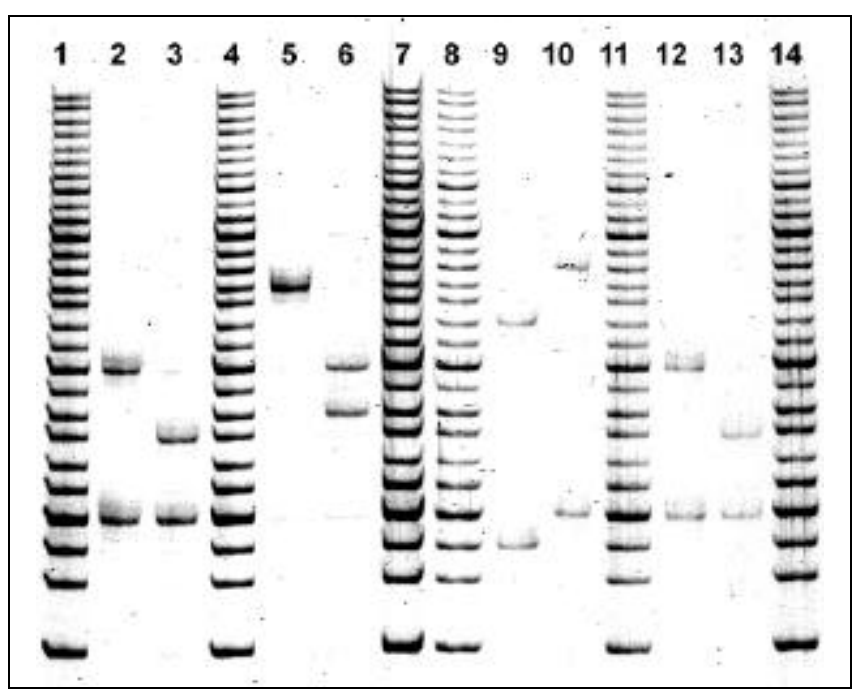

Figure 3. Fluorescent D1S80 typing using SYBR Green I staining. This D1S80 typing gel was stained with SYBR Green I following electrophoresis. Lanes 1-7 contain one-third $(1 \mu \mathrm{L})$ of the standard loading of allelic ladder standard or amplified PCR product. Lanes 8-14 contain one-tenth of the standard amount of sample or ladder. 
and produced very clean blots. A standard paternity test blot with $1 \mu \mathrm{g}$ of DNA per lane showed strong signals four hours after substrate addition (data not shown). Chemifluorescent blots can also be stripped and reprobed effectively without contamination in subsequent hybridizations (data not shown).

\section{D1S80 Typing Using SYBR Green Staining}

The SYBR Green I staining method detected as little as one-tenth of the standard sample loading volume recommended for silver staining protocols (Figure 3). No destaining was required before detection. However, if necessary, the modest background can be reduced by washing the gel in distilled water before scanning. The sensitivity of this detection method allows reagents to be conserved, including the allelic ladder standards that serve as typing reference bands.

\section{Multiplex STR Analysis}

The two strands of the TH01 locus frequently separate

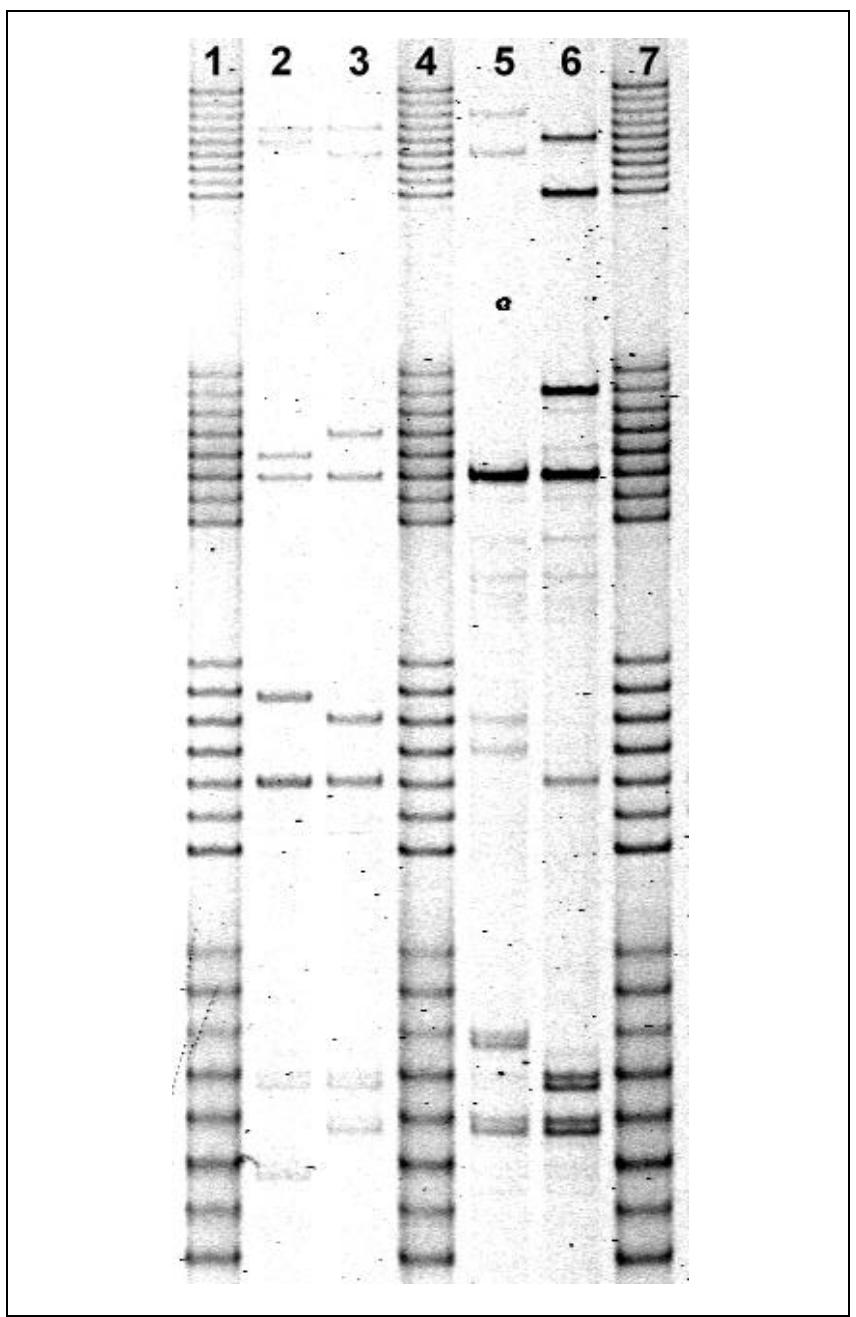

Figure 4. CTTv fluorescent multiplex STR typing. Five to ten nanograms of DNA extracted from four different blood stains were amplified simultaneously at the CSF1PO, TPOX, TH01 and vWA loci, using the GenePrint Fluorescent Multiplex STR system. Lanes 1, 4 and 7 contain allelic ladders for the four loci which serve as typing reference bands. Lanes 2, 3, 5 and 6 contain typing samples 1-4, respectively. Samples 1 and 2 were amplified from $10 \mathrm{ng}$ of extracted DNA with $1.5 \mu \mathrm{g}$ of BSA added to these reactions to improve yield. Samples 3 and 4 were amplified from $5 \mathrm{ng}$ of extracted DNA, without added BSA. 
under standard denaturing conditions (15). Consequently, the PCR products amplified at this locus frequently appear as a "doublet" pattern by silver (15) or SYBR Green staining (11), complicating interpretation of test results. To alleviate this, we used the GenePrint Fluorescent STR Multiplex System CTTv to type four extracted blood stain DNA samples at the CSF1PO, TPOX, TH01 and vWA loci. One primer for each locus is 5'-end labeled with fluorescein. A mixture of fluorescein-labeled allelic ladders for all four loci serves as typing reference bands (Figure 4). We observed a two-band pattern at the vWA locus. This is most likely caused by the terminal transferase activity of the Taq DNA polymerase (15).

\section{DISCUSSION}

Like chemiluminescence, chemifluorescence is based on the affinity binding of an enzyme-conjugated probe to the target molecule. In chemifluorescence, each alkaline phosphatase conjugate can generate many fluorochrome molecules, continually increasing the signal intensity. These fluorochromes emit light only during exposure to the excitation light (i.e., within $10 \mathrm{~ns}$ of absorption), but a single fluorochrome can be excited repeatedly and emit light each time. Thus, a fluorescent blot can be scanned repeatedly without damaging the fluorochrome. If the signal on a blot is weak after the first scan, the substrate incubation can be continued up to $24 \mathrm{~h}$ to develop additional signal before re-scanning. A chemiluminescent molecule, in contrast, is autonomously luminous. No excitation is required, but the luminescence peaks within minutes of substrate addition and decays over a few hours. If a chemiluminescent signal is weak, an additional, longer film exposure is required to collect additional signal. These multiple film exposures are costly at about one dollar per film sheet compared to 3-4 cents per page to print a digitized chemifluorescent image.

Unlabeled PCR products can be detected after electrophoresis sensitively and economically with SYBR Green I. This fluorescent stain has been shown to detect as little as 20 pg of DNA in gels (13). It is ten times more sensitive than ethidium bromide for detection of DNA in acrylamide gels on the FluorImager SI (Peter McNamara, personal communication). SYBR Green is also "essentially non-mutagenic" by the Ames assay (2). This single-step method is much faster than silver staining methods, which may require $90 \mathrm{~min}$ or more $(1,3)$. This method costs less than 50 cents per gel, compared to five dollars per gel for commercial silver staining systems.

Fluorescent end-labeled PCR products provide cleaner, more easily interpreted images than those obtained with staining methods. Since only one primer of each PCR primer pair is labeled with fluorescein, only one product strand is visible at each locus. This eliminates the allelic doublet patterns observed in stained gels run under denaturing conditions (15). End-labeled primers also reduce the problem of artifactual bands that may amplify in multiplex reaction cocktails (9). PCR fragments resulting from extension of unlabeled primers during PCR would be undetectable.

The FluorImager system provides a common analysis platform suitable for detecting and quantitating many of the genetic typing tests used in forensic analysis or parentage testing. Fluorescence scanning offers advantages of high sensitivity, direct quantitation, reagent stability, simple protocols and low reagent costs. The instrument offers rapid, sensi- tive scanning, and the analysis software tools allow reproducible and quantitative analysis of PCR products or RFLP blots. This versatility makes it an attractive instrument platform for genetic typing.

\section{ACKNOWLEDGMENTS}

Dr. Marcia Eisenberg (Roche Biomedical Laboratory) kindly provided E.S.M. with precast gels for the D1S80 studies. Deborah Smead and Luigi Vitiritti provided technical assistance in initial development of chemifluorescent methods for RFLP analysis.

\section{REFERENCES}

1.Bassam, B.J., G. Caetano-Anolles and P.M. Gresshoff. 1991. Fast and sensitive silver staining of DNA in polyacrylamide gels. Anal. Biochem. 196:80-83.

2.Berg, C.M., S. Rooney, X. Liu, F. LaBanca, S. Pickett, X. Jin and V. L. Singer. 1996. Effects of SYBR Green I stain on electrophoretic mobility of supercoiled and open circular DNA. In Abstracts of the 96 th General Meeting of the American Society for Microbiology 1996. H-135.

3.Boulikas, T. and R. Hancock. 1981. A highly sensitive technique for staining DNA and RNA in polyacrylamide gels using silver. J. Biochem. Biophys. Methods 102:53-58.

4.Edwards, A., A. Civitello, H.A. Hammond and C.T. Caskey. 1991. DNA typing and genetic mapping with trimeric and tetrameric tandem repeats. Am. J. Hum. Genet. 49:746-756.

5.Eisenberg, A.J., P. Gibson, S. Nandi and L. Wang. 1992. The development and implementation of a HaeIII based RFLP system for parentage testing in Texas, p. 163-180. In Proceedings from The Second International Symposium on Human Identification 1991. Promega Corporation, Madison, WI.

6.Grimberg, J., S. Nawoschik, L. Belluscio, R. McKee, A. Turck and A. Eisenberg. 1989. A simple and efficient non-organic procedure for the isolation of genomic DNA from blood. Nucleic Acids Res. 17:8390.

7.Kasai, K., Y. Nakamura and R. White. 1990. Amplification of a variable number of tandem repeats (VNTR) locus (pMCT118) by PCR and its application to forensic science. J. Forensic Sci. 35:1196-1200.

8.Liang, B.C., E. Van Gelder, R. Loder, P. Davis, D. Barker and R. Johnston. 1993. Rapid high-sensitivity laser-excited fluorescent gel scanner. FASEB J. 7:A1132.

9.Lins, A.M., C.J. Sprecher, C. Puers and J.W. Schumm. 1996. Multiplex sets for the amplification of polymorphic short tandem repeat loci-silver stain and fluorescent detection. BioTechniques 20:882-889.

10.Mansfield, E.S., J.M. Worley, S.E. McKenzie, S. Surrey, E. Rappaport and P. Fortina. 1995. Nucleic acid detection using non-radioactive labelling methods. Mol. Cell. Probes 9:145-156.

11.Mansfield, E.S., J.M. Worley, P. Zimmerman, M.S. Ma and S. Lee. 1994. Fluorescent detection of Southern blots and PCR-based genetic typing tests. Am. J. Hum. Genet. 56:A1131.

12.Schaap, A.P., H. Akhavan and L.J. Romano. 1989. Chemiluminescent substrates for alkaline phosphatase: application to ultrasensitive enzymelinked immunoassays and DNA probes. Clin. Chem. 35:1863-1864.

13.Singer, V.L., S. Jin, D. Ryan and S. Yue. 1994. SYBR Green dyes: ultrasensitive stains for detection of DNA and RNA in electrophoretic gels. Biomed. Products 19:68-72.

14.Southern, E.M. 1975. Detection of specific sequences among DNA fragments separated by gel electrophoresis. J. Mol. Biol. 98:503-517.

15.Sprecher, C.J., C. Puers, A.M. Lins and J.W. Schumm. 1996. General approach to analysis of polymorphic short tandem repeat loci. BioTechniques 20:266-276.

16.Waye, J.S., L.A. Presley, B. Budowle, G.G. Shuttler and R.M. Fourney. 1989. A simple and sensitive method for quantifying human genomic DNA in forensic specimen extracts. BioTechniques 7:852-855.

17.Wyman, A.R. and R. White. 1980. A highly polymorphic locus in human DNA. Proc. Natl. Acad. Sci. USA 77:6754-6758.

Address correspondence to Jennifer Worley, Molecular Dynamics, Inc., 928 E. Arques Avenue, Sunnyvale, CA 94086, USA. Internet:jworley@mdyn.com 